\title{
Scaling Properties of Biologically Active Scalar Concentration Fluctuations in the Atmospheric Surface Layer over a Managed Peatland
}

\author{
Matteo Detto • Dennis Baldocchi • Gabriel G. Katul
}

Received: 13 October 2009 / Accepted: 25 May 2010 / Published online: 16 June 2010

(C) The Author(s) 2010. This article is published with open access at Springerlink.com

\begin{abstract}
The higher-order scalar concentration fluctuation properties are examined in the context of Monin-Obukhov similarity theory for a variety of greenhouse gases that have distinct and separate source/sink locations along an otherwise ideal micrometeorological field site. Air temperature and concentrations of water vapour, carbon dioxide and methane were measured at high frequency $(10 \mathrm{~Hz})$ above a flat and extensive peat-land soil in the San Joaquin-Sacramento Delta (California, USA) area, subjected to year-round grazing by beef cattle. Because of the heterogeneous distribution of the sources and sinks of $\mathrm{CO}_{2}$ and especially $\mathrm{CH}_{4}$ emitted by cattle, the scaling behaviour of the higher-order statistical properties diverged from predictions based on a balance between their production and dissipation rate terms, which can obtained for temperature and $\mathrm{H}_{2} \mathrm{O}$ during stationary conditions. We identify and label these departures as 'exogenous' because they depend on heterogeneities and nonstationarities induced by boundary conditions on the flow. Spectral analysis revealed that the exogenous effects show their signatures in regions with frequencies lower than those associated with scalar vertical transport by turbulence, though the two regions may partially overlap in some cases. Cospectra of vertical fluxes appear less influenced by these exogenous effects because of the modulating role of the vertical velocity at low frequencies. Finally, under certain conditions, the presence of such exogenous factors in higher-order scalar fluctuation statistics may be 'fingerprinted' by a large storage term in the mean scalar budget.
\end{abstract}

Keywords Carbon dioxide $\cdot$ Concentration fluctuations $\cdot$ Methane $\cdot$ Peatland · Similarity theory $\cdot$ Storage flux

\footnotetext{
M. Detto $(\bowtie) \cdot$ D. Baldocchi

ESPM, University of California at Berkeley, Berkeley, CA 94720, USA

e-mail: mdetto@berkeley.edu; matteo.detto@polimi.it
}

G. G. Katul

Nicholas School of the Environment, Duke University, Durham, NC 27708-0328, USA

G. G. Katul

Department of Civil and Environmental Engineering, Pratt School of Engineering,

Duke University, Durham, NC 27708, USA 


\section{Introduction}

The problem of passive scalar transport by turbulence remains at the frontier of turbulence research (Shraiman and Siggia 2000). In the atmosphere, scalar transport is complicated by additional processes such as dynamic and heterogeneous ground sources and sinks, thermal stratification, modulations by mesoscale processes and entrainment, to name a few. Towerbased observations, often carried out in the so-called atmospheric surface layer (ASL), have exponentially proliferated over the past two decades to permit continuous and long term measurements of velocity and scalar statistics (Baldocchi 2003). What is lacking now is a theoretical framework to interpret their variability and scaling properties (Finnigan 2008). The main factors impeding theoretical developments can be categorically lumped into: (1) 'endogenous' factors because scalar transport dynamics is inherently rich in scales of variability even in the absence of any spatial and temporal variability in scalar source strength, and (2) 'exogenous' factors because heterogeneity and unsteadiness are introduced in the boundary conditions, whether at the top of the atmospheric boundary layer, such as entrainment, at the ground, or from a distant source.

In the absence of such 'exogenous' factors, the classical Monin-Obukhov Similarity Theory (MOST, Monin and Obukhov 1954), can be used to explain key features of the bulk flow statistics of the mean scalar concentration (Stull 1988; Garratt 1992), hereafter, referred to for simplicity as 'global' statistics because they describe statistics computed over a long averaging period such as $1 \mathrm{~h}$. In MOST, the global statistics can be canonically described by the distance from the ground and by the surface forcing (friction velocity and scalar surface fluxes). By virtue of the mean scalar continuity equation, the vertical fluxes should remain constant with height for stationary and planar-homogeneous high Reynolds number and Peclet number flows with no subsidence. However, in many ecosystems, horizontal and vertical advection, surface heterogeneity, unsteadiness and mesoscale effects are known to contribute to the failure of MOST and its use in interpreting trace gas fluxes (Katul et al. 1995; McNaughton and Laubach 1998; Asanuma and Brutsaert 1999; De Bruin et al. 1999; Lamaud and Irvine 2006; Detto et al. 2008). Moreover, in the very stable boundary layer, the scaling properties of these global statistics are complicated by other non-turbulent mechanisms (Mahrt 1989; Mahrt et al. 1998). The latter violations are commonly associated with atmospheric phenomena such as gravity waves (Finnigan et al. 1984; Sun et al. 2004) or canopy waves (Cava et al. 2004) and low-level jets (Nappo 1991; Prabha et al. 2007). Very stably stratified flows may not be entirely turbulent. Hence, the effects of terrain irregularities and scalar source distributions are not rapidly blended or smeared, thereby casting doubts on the applicability of MOST. If sources are localized or sparse, a decrease in the diffusive properties of turbulence may result in a single plume being coherently advected long distances before being distorted and dissipated. Meandering streams of air, caused by continuous shifts in wind direction, allow random plumes to occasionally arrive at the point of measurement resulting in complex and highly non-stationary patterns in scalar concentration time traces. Intermittent turbulence at night is also a complicating factor in measuring ecosystem respiration with confidence (Cava et al. 2004; Acevedo et al. 2007).

For scalar turbulent fluctuations at the so-called intermediate time scales, the 'knowledge gap' is also wide. The term 'intermediate' refers to scales smaller than the usual 'turbulent averaging interval' of 20-60 min, but larger than the inertial scales. These intermediate scales are crucial because they are linked with eddy sizes that are comparable to the scales of source heterogeneity and hence may dynamically interact with them (Detto et al. 2008). Early studies already recognized that low frequencies of longitudinal and lateral wind spectral functions do not scale with MOST variables because they are modulated by large-scale (inactive) motion 
that may be independent of surface drag (Townsend 1961). This conclusion, which may be extended for scalar spectra, is now the subject of re-interpretation by a number of studies that demonstrate entrainment processes (i.e. an exogenous effect) can play a crucial role in ASL scalar transport (Katul et al. 2008).

Collectively, these findings begin to suggest that the global statistics of scalar concentration fluctuations may be decomposed into a local component that describes the equilibrium state (and most likely tracks MOST scaling) and a non-local component introduced by exogenous factors that disturbs this local equilibrium state.

The implication of this hypothesis on scalar variances and higher-order scalar concentration moments can benefit from field experiments, and one such experiment is the subject of this investigation. Turbulence properties have been collected in the ASL above a flat and extended, managed peatland ecosystem. The main novelty of this field experiment is that sources and sinks of various bio-physically interactive scalars (such as temperature, $\mathrm{H}_{2} \mathrm{O}, \mathrm{CO}_{2}$ and $\mathrm{CH}_{4}$ ) remain markedly different (i.e. different exogenous factors), especially in the presence of moving cattle, yet they are being transported by the same flow regime above a flat plateau (similar endogenous flow dynamics). Water vapour and air temperature fluctuations primarily arise from latent and sensible heat exchanges between land and atmosphere. An air mass can also be advected from an upwind water body (such as the San Joaquin River, which is just $5 \mathrm{~km}$ from the experimental site) encoding a different partitioning of the surface energy fluxes compared to that over the land surface. Over vegetated areas, exchanges of water vapour are correlated with carbon dioxide as the plant system assimilates $\mathrm{CO}_{2}$ and transpires $\mathrm{H}_{2} \mathrm{O}$. This is not necessarily true for ecosystem respiration, since decomposition in organic soils is associated with bacterial activity. Additional sources of $\mathrm{CO}_{2}$ may be non-local to the immediate underlying surface due to long-distance transport from urban areas, for example from refineries and power plants that exist in the upwind airshed of this site, or in this specific case, from cattle respiration. The production of methane in the soil is related to anoxic and highly reducing conditions (Cicerone and Oremland 1988), which are formed in areas where ground water is elevated to the surface or where the surface has been flooded by irrigation. In drained peatlands, these differences can be amplified because of the intensive livestock farming, including large emissions of methane from cattle.

Using single-point statistics to explore these non-local effects originating far from the measuring point becomes challenging. One 'thorny' issue here is that the ergodicity hypothesis cannot be readily used because one realization (or a time series run) may not contain a large enough number of such non-local events to represent their ensemble behaviour (Katul et al. 2003). This is even more true if the microclimatic conditions (wind direction, air diabatic conditions etc.) or the sources are not stationary. However, departures from MOST scaling (that predicts expected ensemble behaviour at the equilibrium state) may provide an order of magnitude estimate on the intensity of these non-local disturbances. The availability of synchronous and multiple scalar time series can further elucidate the contribution of various exogenous factors. Most studies to date have focused on relatively few scalars, mainly temperature and humidity. Recently, advances in tunable diode laser spectroscopy are now producing sensors for other radiative greenhouse gases, such as methane (Baer et al. 2002; Hendriks et al. 2008), known to possess complex footprints and variable source production rates attributed to heterogeneous soil microbial process, or in this case, emissions from cattle that form a heterogeneous and dynamic source.

These scalars are generally classified as passive, if they exert no influence (such as buoyancy) on the velocity, unlike temperature and to a less extent water vapour, and they have no mass inertia in contrast to rain drops or wind dispersed seeds. Chemically reactive 
scalars possessing a large Damköhler number (ratio of the reaction to turbulent dispersion time scales) will not be considered here.

Our overarching objective will be to assess the relative importance of endogenous and exogenous factors on a multi-scalar fluctuation statistical ensemble with different source and sink locations and degrees of stationarity. Higher-order statistics are known to be sensitive to exogenous effects when compared to lower-order moments (e.g. mean concentration). If these exogenous factors primarily affect a region of the scalar spectrum that does not necessarily overlap with the scalar turbulent flux time scales (e.g. comparable to the integral time scales of vertical velocity), it is possible to 'filter out' these intermediate or low frequency modulations, as was done by Vickers and Mahrt (2006), and explore their statistics separately.

\section{Theory}

We interpret the measurements of scalar turbulence statistics through prognostic equations for the mean and fluctuating scalar concentration, $s$, in the ASL, as given by Stull (1988):

$$
\begin{gathered}
\frac{\partial \bar{s}}{\partial t}+\bar{u}_{i} \frac{\partial \bar{s}}{\partial x_{i}}=-\frac{\partial \overline{u_{i}^{\prime} s^{\prime}}}{\partial x_{i}} \\
\frac{\partial s^{\prime}}{\partial t}+\bar{u}_{i} \frac{\partial s^{\prime}}{\partial x_{i}}+u_{i}^{\prime} \frac{\partial \bar{s}}{\partial x_{i}}+u_{i}^{\prime} \frac{\partial s^{\prime}}{\partial x_{i}}=v_{s} \frac{\partial^{2} s^{\prime}}{\partial x_{i}^{2}}+\frac{\partial \overline{u_{i}^{\prime} s^{\prime}}}{\partial x_{i}}
\end{gathered}
$$

where the overbar represents time averaging, $x_{i}=\left(x_{1}, x_{2}, x_{3}\right)$ or $x, y, z$ are the longitudinal, lateral, and vertical directions, respectively, $u_{i}=\left(u_{1}, u_{2}, u_{3}\right)$ or $u, v, w$ are the three velocity components along directions $x_{i}$, primed quantities represent excursions from the mean state due to turbulence and other non-local phenomena, and $v_{s}$ is the molecular diffusivity. Multiplying Eq. $1 \mathrm{~b}$ by $n s^{\prime n-1}$, where $n$ is the order of the scalar moment being analyzed, and applying the usual Reynolds's averaging operator, the budget equation of any $n^{\text {th }}$-moment can be derived as

$$
\underbrace{\frac{\partial \overline{s^{\prime n}}}{\partial t}}_{I}+\underbrace{\bar{u}_{i} \frac{\partial \overline{s^{\prime n}}}{\partial x_{i}}}_{I I}=-\underbrace{n \overline{u_{i}^{\prime} s^{\prime n-1}} \frac{\partial \bar{s}}{\partial x_{i}}}_{I I I}+\underbrace{\frac{\partial \overline{u_{i}^{\prime} s^{\prime n}}}{\partial x_{i}}}_{I V}+\underbrace{n \overline{s^{\prime n-1}} \frac{\partial \overline{u_{i}^{\prime} s^{\prime}}}{\partial x_{i}}}_{V}+\underbrace{n \varepsilon_{s, n}}_{V I}
$$

where these terms take on their usual meaning in the Reynolds-averaged equations with term $I$ being the local rate of change, term $I I$ being the mean advective rate of change, term $I I I$ being the production term by the mean flow, terms $I V$ and $V$ being the turbulent transport terms, and term $V I, \varepsilon_{s, n}=-v_{s} \overline{s^{\prime n-2}\left(\partial s^{\prime} / \partial x_{i}\right)^{n}}$, is the mean dissipation rate of the $n^{\text {th }}$-moment. Note that term $V$ only appears for $n>2$.

\subsection{Simplifications}

For the 'idealized' case of steady and planar homogenous flow with no subsidence $\left(\bar{u}_{3}=0\right)$, terms containing $\frac{\partial}{\partial t}, \frac{\partial}{\partial x}, \frac{\partial}{\partial y}$ and $\bar{u}_{3} \frac{\partial}{\partial x_{3}}$ can be neglected. With these simplifications, Eqs. $1 \mathrm{a}$ and 2 reduce to: 


$$
\begin{gathered}
\frac{\partial \overline{w^{\prime} s^{\prime}}}{\partial z}=0, \\
n \frac{\partial \overline{w^{\prime} s^{\prime n-1}} \frac{\partial \bar{s}}{\partial z}}{=}=\frac{\partial \overline{w^{\prime} s^{\prime n}}}{\partial z}+n \varepsilon_{s, n} . \\
I I I
\end{gathered}
$$

Equation $3 \mathrm{a}$ affirms that these idealized conditions are necessary for the formation of a so-called constant-flux layer in the ASL (Kaimal and Wyngaard 1990) as assumed by MOST. Hence, for first-order statistical moments $(n=1)$, the transport term $I V$ in Eq. $3 b$ can be neglected. Whether this transport term $I V$ can be neglected for $n>1$ remains questionable, and subject to investigation here. For the temperature second-order budget $(n=2)$, it has been observed that this term may be small for some cases (e.g., Wyngaard and Cote 1971; Bradley et al. 1981; Zhang and Park 1999, where the transport terms were actually measured). If so, the budget in Eq. $3 \mathrm{~b}$ may be reduced to a balance between production and dissipation terms (terms III and VI). However, other studies (e.g., Kiely et al. 1996; Hsieh and Katul 1997) have reported dissipation values smaller than production, especially in the convective ASL, supporting the hypothesis of Monji (1973) that scalar variance is transported from the surface to regions of the ABL where production is absent. The importance of these fluxtransport terms for statistical moments greater than two $(n>2)$ are rarely considered. Antonia et al. (1982) found for near-neutral conditions, the transport term becomes small compared with production for $n=3$.

\subsection{Closure Assumptions}

Standard ASL scaling is often represented as $\frac{\partial \bar{s}}{\partial z} \sim \frac{s_{*}}{\ell_{s}}, \varepsilon_{s, n} \sim \frac{\overline{s^{\prime n}}}{\tau_{s}}$, where $s_{*}=\overline{w^{\prime} s^{\prime}} / u_{*}$ is a characteristic scalar concentration formed from variables that control the lower boundary conditions on the flow, and $\ell_{s}$ and $\tau_{s}$ are characteristic length and time scales. The production balancing dissipation rate arguments result in the $n^{\text {th }}$-moment scaling as:

$$
\left|\overline{\overline{s^{\prime n}}}\right| \sim s_{*} \frac{\tau_{s}}{\ell_{s}}=\frac{s_{*}}{v_{s}} .
$$

Note that $\ell_{s}$ and $\tau_{s}$ can be combined to form a characteristic velocity scale, $v_{s}$, which is expected to vary with atmospheric stability and follow MOST scaling in the absence of exogenous effects. In particular $v_{s}^{-1} u_{*}$ should be a constant in neutral conditions and $v_{s}^{-1} u_{*} \propto(-z / L)^{-2 / 3}$ in convective conditions, where $L$ is the Obukhov length. The choice of this velocity scale is not unique. For example, McNaughton (2006) proposed an alternative velocity scale to $u_{*},(0.4 z \varepsilon)^{1 / 3}$, presumably to include modulations from outer-layer processes (e.g. exogenous effect). The scalar velocity $v_{s}$, normalized by this velocity scale, has the advantage of removing the $-2 / 3$ scaling with stability as shown elsewhere (von Randow et al. 2006). Because we seek to represent the 'equilibrium' state by MOST and analyze perturbations from this state, we use MOST scaling for $v_{s}$.

\section{Materials and Methods}

\subsection{Site Description}

The wind velocity and scalar turbulence measurements were conducted on Sherman Island in California, USA (38.0373N, 121.7536W; elevation -12.1 m) starting from 5 April 2007 


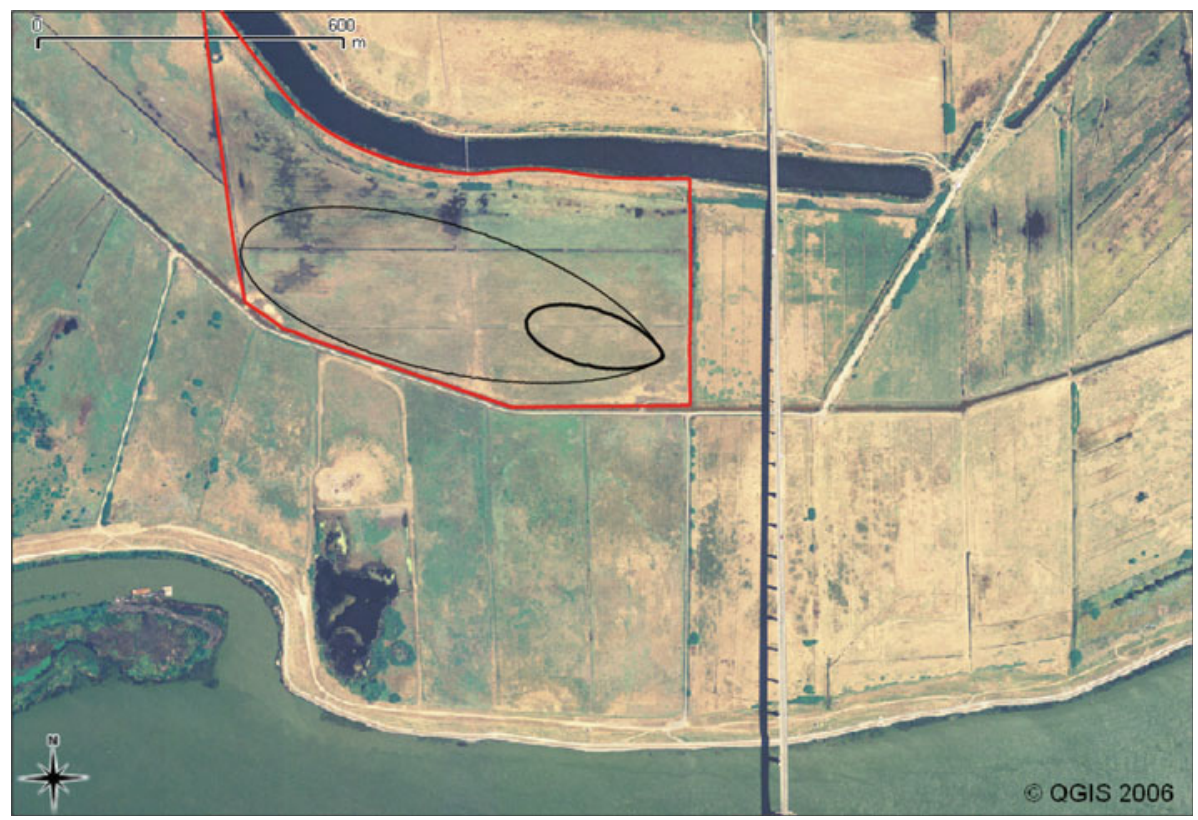

Fig. 1 Aerial photograph of the Sherman Island experimental site in the San Joaquin Delta River (California). Contour lines enclose the $90 \%$ of a typical daytime footprint (thick solid line) and nighttime footprint (thin line). The flux footprint was evaluated using an approximate analytical model (Hsieh et al. 2000) extended to two-dimensions by Detto et al. (2006). Red lines delimit the cows' paddock

until 31 August 2009. The field site is located on the west side of the Sacramento-San Joaquin Delta and is on land managed by the California Department of Water Resources. A map of the area is provided in Fig. 1.

The wind directions are regular and generally prevail from the north-west, the result of the field site being near the Carquinez Straits and at the confluence of the Sacramento and San Joaquin rivers. This geographic configuration funnels fairly strong winds between the coast and the interior valley. Nocturnal winds are lighter, but are still of notable magnitude; friction velocity fell below $0.1 \mathrm{~m} \mathrm{~s}^{-1}$ only $12 \%$ of the time. The relatively flat land was prominently covered by perennial pepper weed (Lepidium latifolium L.) in the immediate fetch of the tower; the weed appeared in mid-March, when new shoots emerged from root buds, and persisted until the end of October. During the study period, the water table was about $0.5 \mathrm{~m}$ deep, rising to $0.3 \mathrm{~m}$ during the rainy season (December-February) and $0.7 \mathrm{~m}$ in summer. Large evapotranspiration was observed during the dry hot summer months (up to $5 \mathrm{~mm}$ per day), because of the ability of the plant roots to tap into the shallow water table. The peat soil respired $\mathrm{CO}_{2}$ at appreciable rates $\left(\approx 5 \mu \mathrm{mol} \mathrm{m}^{-2} \mathrm{~s}^{-1}\right)$, and in addition, $\mathrm{CO}_{2}$ is produced by cattle respiration, and detected by the tower when the cattle are relatively close and in large numbers.

However, the presence of the cattle seems to affect primarily methane concentrations. Despite the soil being a modest source or sometimes a small sink, mean vertical fluxes can exceed $1,000 \mathrm{nmol} \mathrm{m}^{-2} \mathrm{~s}^{-1}$. Dry cows (non lactating), in fact, can emit $3.5 \times 10^{3} \mu \mathrm{mol}$ of $\mathrm{CO}_{2}$ cow ${ }^{-1} \mathrm{~s}^{-1}$, and $2.2 \times 10^{5} \mu \mathrm{mol}$ of $\mathrm{CH}_{4} \mathrm{cow}^{-1} \mathrm{~s}^{-1}$ (Shaw et al. 2007). Considering the extension of the paddock is $0.38 \mathrm{~km}^{2}$ and a total of one hundred cows, the average $\mathrm{CO}_{2}$ and $\mathrm{CH}_{4}$ fluxes produced by cows per unit area are approximately $1 \mu \mathrm{mol} \mathrm{m}^{-2} \mathrm{~s}^{-1}$ and 
$60 \mathrm{nmol} \mathrm{m}{ }^{-2} \mathrm{~s}^{-1}$, respectively. Estimates of soil emission, made when the cows were not present in the field range between -4 and $10 \mathrm{nmol} \mathrm{m}^{-2} \mathrm{~s}^{-1}$, confirmed also by chamber measurements (Teh, pers. commun. 2009) taken within the local fetch of the tower (200-300 m).

Furthermore, the surrounding fields were temporary flooded and strong methane emissions were observed from the irrigation drains along the perimeters of these irrigated pastures, as verified by independent chamber measurements (Fig. 1). A large restored wetland downwind may also contribute substantially to the regional methane concentrations.

\subsection{Instrumental Set-Up}

Fluctuations of the velocity components and speed of sound were measured with a Windmaster sonic anemometer (Gill Instruments). An infrared open path gas analyzer (Li-Cor 7500) was used to sample $\mathrm{CO}_{2}$ and $\mathrm{H}_{2} \mathrm{O}$ density fluctuations, while a new generation of infrared laser absorption spectrometers (Baer et al. 2002) was used to measure $\mathrm{CH}_{4}$ concentrations. The sampling air was drawn at a flow rate of 35 standard litres per min by a scroll pump (Edwards ESDP 30) through a 5-m tube (inner diameter $0.0064 \mathrm{~m}$ ) into a sample cell $\left(408 \times 10^{-6} \mathrm{~m}^{3}\right)$ operating at a pressure of 142 Torr. The sonic anemometer was positioned at $3.2 \mathrm{~m}$ above the ground, the inlet tube for methane was located about the same height and $0.20 \mathrm{~m}$ east of the sonic anemometer, while the Li-Cor analyzer was located $0.20 \mathrm{~m}$ west, just below the sonic anemometer. All the instrument signals were sampled at a frequency of $10 \mathrm{~Hz}$ and stored for later processing.

During the months of July and August, 2009, a second tower was added and equipped with an identical sonic anemometer and open-path gas analyzer at $1.5 \mathrm{~m}$ height.

\subsection{Data Processing}

A de-spiking algorithm was developed to detect and remove spikes in velocity and scalar concentration series. Bulk statistics were computed as follows: a de-spiking, the time series were divided into overlapping blocks of $2^{14}$ data points (approximately $27 \mathrm{~min}$ ), one every $15 \mathrm{~min}$. Even though no relevant topography was present at the site, small tilt angles cannot be avoided in the positioning of the sonic anemometer. For each block, two coordinate rotations were employed to align the $u$ component to the mean wind direction by setting the mean lateral and vertical velocities to zero. Time lag responses of each gas analyzer were evaluated using the maximum cross-correlation method (relative to the vertical velocity); these time lags were approximately constant at 0.1 and $1.1 \mathrm{~s}$ for $\mathrm{H}_{2} \mathrm{O} / \mathrm{CO}_{2}$ and $\mathrm{CH}_{4}$, respectively. The air temperature fluctuations were computed from fluctuations in the speed of sound after removing crosswind and humidity effects (Schotanus et al. 1983; Kaimal and Gaynor 1991). The $\mathrm{H}_{2} \mathrm{O}$ and $\mathrm{CO}_{2}$ scalar densities (mol of gas per volume of air) were adjusted to take into account air density fluctuations (due to temperature and water vapour concentration variations) using the formulation derived in Detto and Katul (2007). The $\mathrm{CH}_{4}$ concentration time series (mol of gas per mol of air) were also adjusted to remove variations in air density due to the transfer of water vapour only, assuming high frequency temperature fluctuations were mostly damped when sampling air through a tube. Hereafter, turbulent fluctuations of temperature, water vapour, carbon dioxide and methane are abbreviated as $T, q, c$ and $m$, respectively.

Once all the statistical moments were computed (means, variances, and covariances along with other higher central moments) for each block, a first screening was employed to remove spurious runs. Data acquired during and immediately after rain events, during fog, common during winter, were removed. Runs with small vertical fluxes were not excluded, unless the 


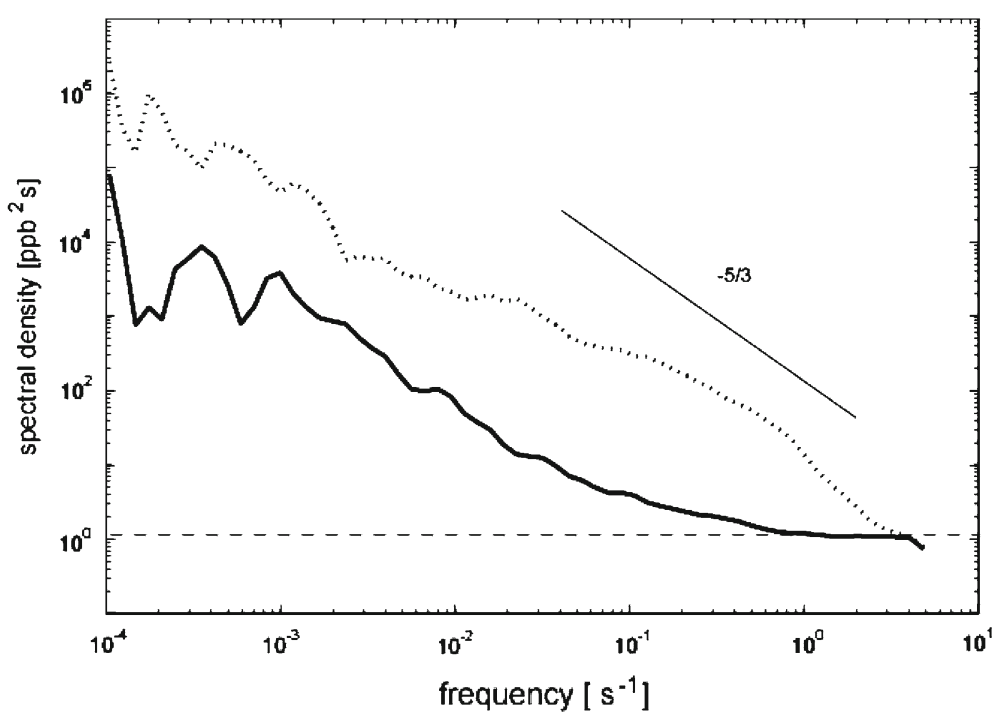

Fig. 2 Wavelet-based spectral densities of methane concentration for two periods of $3 \mathrm{~h}$ recorded at the same day during low scalar variance (standard deviation equal to $\pm 10.6 \mathrm{ppb}$, solid line), and high scalar variance $(\mathrm{SD} \pm 57.8 \mathrm{ppb}$, dotted line $)$ due to the passage of cows in the proximity of the tower. Horizontal dashed line represents a white-noise spectrum with a standard deviation of $6 \mathrm{ppb}$. The $-5 / 3$ power-law scaling is also shown for reference

tracer contained significant high frequency white noise. This clarification becomes important in the case of methane, because small mean vertical fluxes were associated with large concentration fluctuations. However, the signal-to-noise level of the methane measurements are small when compared to the other tracers. The white-noise level was evaluated by spectral analysis according to the asymptotic value at the highest frequencies during periods of low scalar variance (usually during the daylight and at high winds). The argument here is that the theoretical spectrum of scalar turbulence should decay as a power law with increasing frequency and hence the highest frequencies are most contaminated by white noise. This is illustrated in the example reported in Fig. 2. The noise standard deviation, evaluated as the area of the spectrum below this asymptotic value, was estimated at $6 \mathrm{ppb}$, a value consistent with the noise level found when running air through a closed loop for $30 \mathrm{~min}$ in operative field conditions.

\subsection{Wavelet Analysis}

Wavelet analyses, routinely used in turbulence studies because of their ability to investigate global and local spectral features of non-stationary time series (Argoul et al. 1989; Katul et al. 2001; Terradellas et al. 2001), were employed here for spectral and cospectral calculations. Percival (1995) showed that the global wavelet spectrum provides a consistent estimation of the true power spectrum of a time series and can be considered an excellent smoother of the Fourier power spectra (Hudgins et al. 1993). In our study, a continuous wavelet transform (complex Morlet) is employed to evaluate the spectral and cospectral functions of long-term observations (1 year) of turbulent velocities and scalars. Details on the theory and implementations of wavelet transform can be found elsewhere (Farge 1992; Torrence and Compo 1998) and are not repeated here. Time scales were transformed into spatial scales using 
Taylor's frozen hypothesis, noting that a necessary condition for Taylor's hypothesis is that $\sigma_{u}^{2} / \bar{u}^{2}$ is smaller than $10 \%$ (see Hsieh and Katul 1997 for detailed discussions). Regions outside the cone of influence of the wavelet were excluded in the computation of global spectra and cospectra, reducing the maximum scale from $27 \mathrm{~min}$ to about $8 \mathrm{~min}$. This reduction leads to an increase in the statistical reliability of any estimate at the intermediate to smaller scales. Recall that Taylor's hypothesis does not apply for time scales much larger than the longitudinal integral time scale (Panofsky and Dutton 1984).

Because a key element in our analysis is the equilibrium state at which production balances the dissipation, estimates of the kinetic energy and scalar dissipation rates are needed. The dissipation rates can be inferred from Kolmogorov's celebrated $-5 / 3$ theory, permitting us to relate velocity spectral densities to the mean kinetic energy dissipation rate, $\varepsilon$, as:

$$
S_{u}(\kappa)=C \varepsilon^{2 / 3} \kappa^{-5 / 3}
$$

where $\kappa$ is the wave-number (inferred from Taylor's hypothesis) and $C \approx 0.52$ is a universal constant for longitudinal velocity. The analogous formulation for the mean scalar dissipation rate $\varepsilon_{s}$ is:

$$
S_{S}(\kappa)=C_{s} \varepsilon^{-1 / 3} \varepsilon_{s} \kappa^{-5 / 3}
$$
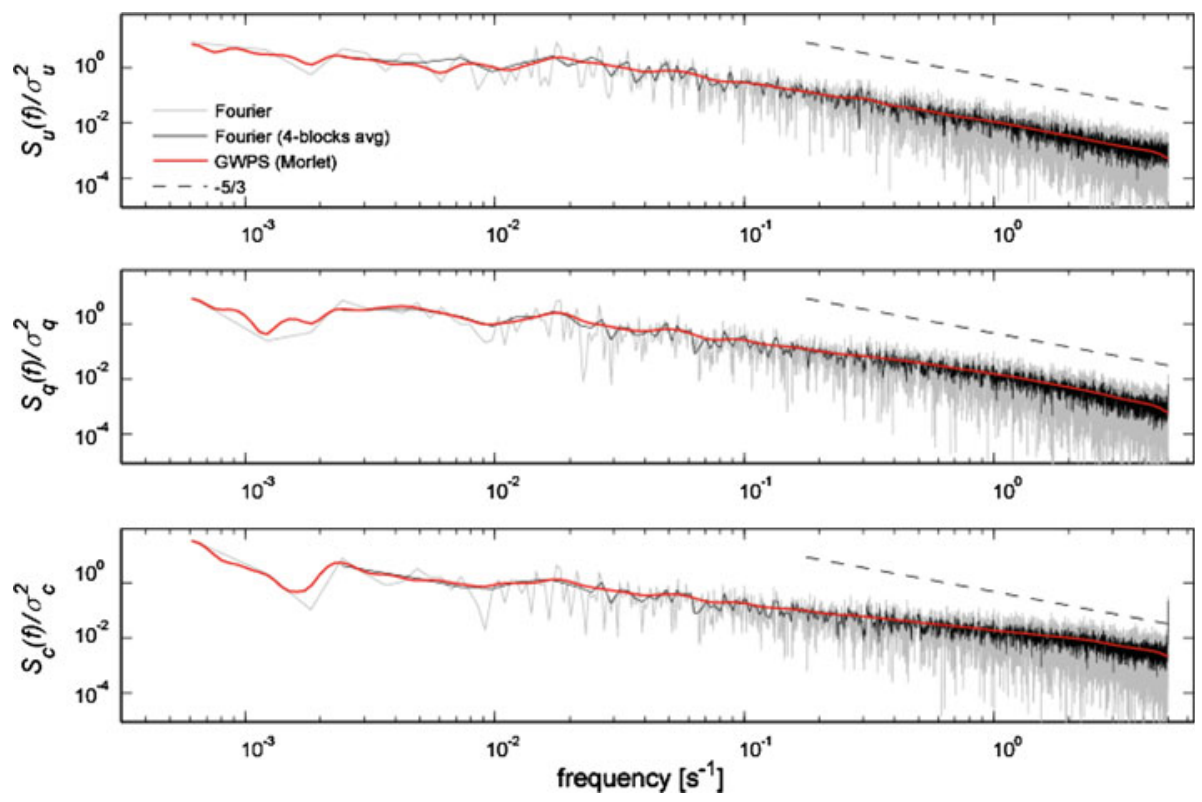

Fig. 3 Comparison between the spectral densities computed from Fourier and Morlet wavelet transforms (with base parameter $\omega_{0}=6$ ) for the normalized longitudinal velocity (top panel), water vapour density (central panel), and carbon dioxide density (bottom panel). Normalization is carried out so that each time series has a zero mean and unit variance. Note that, as the Fourier spectrum is smoothed (using block averaging), it approaches the global wavelet spectrum. The $-5 / 3$ power law is shown for reference. The spectral density (energy per unit of frequency) is evaluated as the mean of the square modulus of the wavelet coefficients for each scale multiply by $C_{\delta} 2 \pi \log (2) / d t$, where the reconstruction factor $C_{\delta}$ is equal to 0.776 . The frequency

of the wavelet transform is computed as the inverse of the corresponding scale multiplied by $\frac{\omega_{0}+\sqrt{2+\omega_{0}^{2}}}{4 \pi}$ to match the Fourier period (Torrence and Compo 1998) 
where $C_{s} \approx 0.8$ is the Obukhov-Corrsin constant (e.g., Verma and Anderson 1984). The wavelet spectrum was shown to be a more robust estimator for $\varepsilon$ and $\varepsilon_{s}$ than its Fourier counterpart (Nichols-Pagel et al. 2008), with Fig. 3 illustrating this point by comparing the wavelet spectra of longitudinal velocity, water vapour and $\mathrm{CO}_{2}$, with their Fourier spectral counterparts. Note that, as the Fourier spectrum is smoothed (using block averaging), it approaches the global wavelet spectrum. Nevertheless, the detection of the inertial subrange scales and concomitant parameters for a large set of atmospheric conditions is not a straightforward task (Antonia and Smalley 2001) as demonstrated later.

\section{Results}

The purpose of our analysis is not to test the production/dissipation equilibrium hypothesis, which is largely discussed elsewhere (Stull 1988; Garratt 1992). Instead, our aim is to show how the scaling $v_{s}^{-1} u_{*}$ can be generalized to higher moments of the scalar fluctuations for a variety of similar and dissimilar trace gases. When computing $v_{s}^{-1} u_{*}$ (using Eq. 4 with $n=2, \ldots, 7)$ and plotting the outcome against the stability parameter $z / L$, remarkable similarities emerge (Fig. 4). Note that the similarity behaviour is not attributed to points collapsing on the power-law decay, as may be inferred from a preliminary inspection of Fig. 4. Rather, it is revealed by the fact that the majority of points are well grouped around the model (better for temperature than for water vapour), with less scatter than expected if all variables were independent. This problem was recently explored by Cava et al. (2008) using
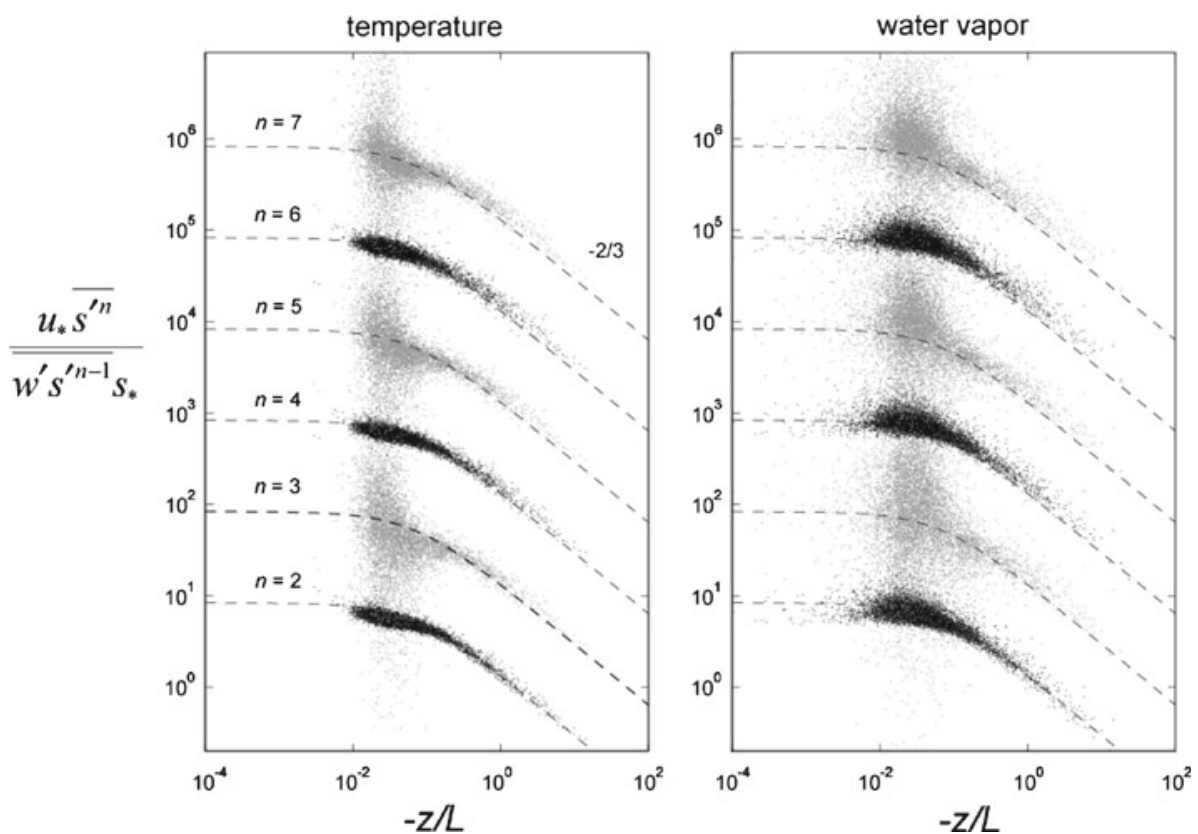

Fig. 4 Normalized higher-order scalar concentration fluctuation moments (also expressed as $v_{S}^{-1} u_{*}$ ), plotted as a function of the atmospheric stability parameter for temperature (left) and water vapour (right). Each increasing moment is shifted upwards by one decade for clarity. The dashed line, $5.6(1-7 z / L)^{-2 / 3}$, is obtained from regression using $n=2$ and air temperature as a scalar 

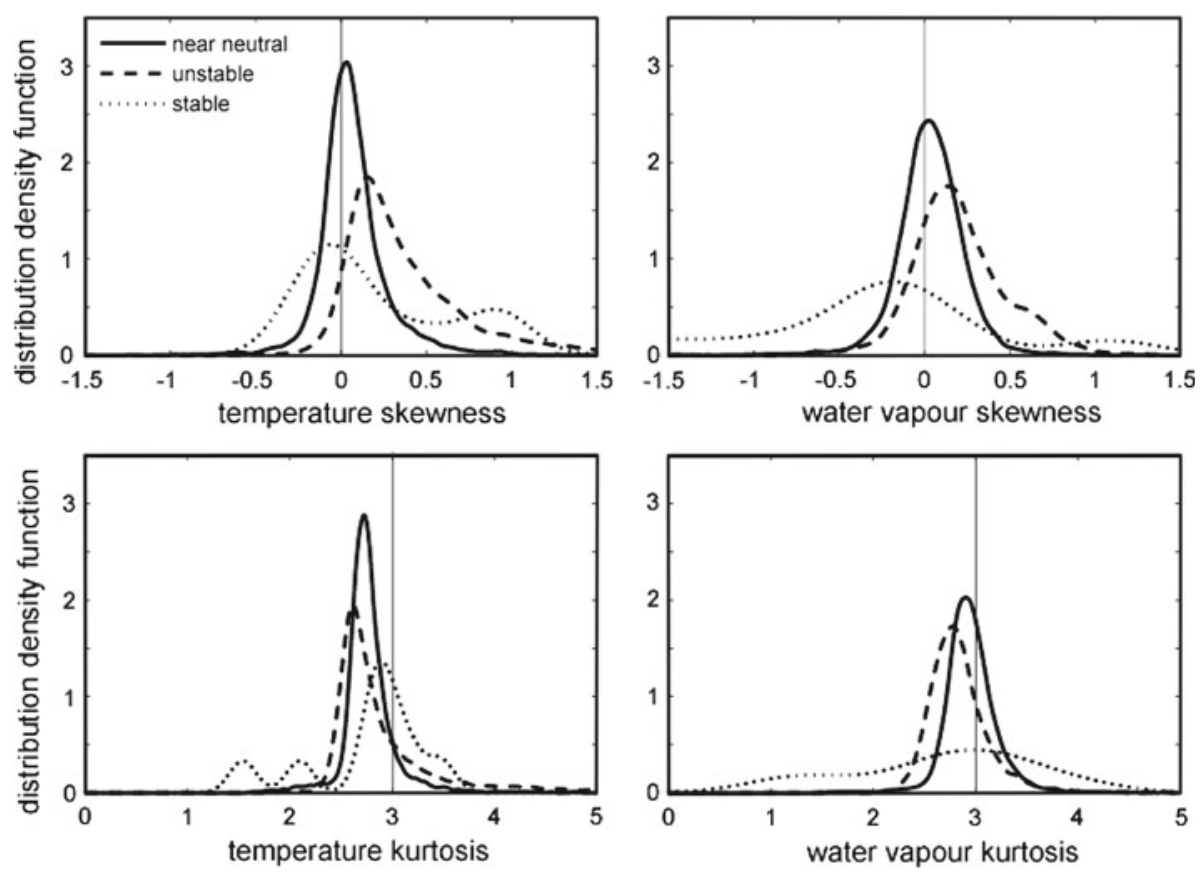

Fig. 5 Probability density functions of skewness (top panels) and kurtosis (bottom panels) of temperature and water vapour fluctuations observed for different atmospheric stability conditions (see Table 1). Vertical grey lines indicate skewness or kurtosis values for a Gaussian distribution

a randomization technique, and who concluded that, in both the near-neutral and convective regimes, the correlations were not mere statistical artefacts. The same test has been applied to the present dataset with analogous results.

During near-neutral conditions, odd-ordered moments, which are a measure of the asymmetry of the scalar concentration fluctuation probability density function (pdfs), are close to zero (Chu et al. 1996) thereby affecting the statistical stability of the ratio in Eq. 4. Considering also that the accuracy of the $n^{\text {th }}$-moment estimate decreases with $n$ (Sreenivasan et al. 1978), the scatter in Fig. 4 does not appear to be large. For increasingly diabatic conditions, the probability density functions become more skewed and the scaling approaches that of the even moments. This argument is shown in Fig. 5, where distributions of skewness and kurtosis are evaluated for the three different stability classes. Note that the modal values of the data are not far from Gaussian. This means that scaling arguments based on the production/dissipation equilibrium hypothesis can be extended to higher-order moments and that the transport terms (known to introduce asymmetry) are either small or more likely assimilated into the local scaling. This idealized equilibrium state forms the basis for formally identifying the endogenous dynamics, with excursions from it attributed to exogenous factors.

To exclude the exogenous effects in Fig. 4, the analysis was conditioned on scalars (temperature and water vapour) that are expected to be more homogeneous and on periods where the storage terms were relatively small, i.e. $\left|z \frac{\partial \bar{s}}{\partial t}\right| \ll\left|\overline{w^{\prime} s^{\prime}}\right|$ and $\left|\frac{\partial s^{\prime 2}}{\partial t}\right| \ll \varepsilon_{s}$. The first constraint is necessary but not sufficient for the onset of a constant-flux layer. This point will be further discussed below. 
Table 1 Statistics (I and III quartiles) of the integral length scale and transport efficiency (correlation coefficient between vertical velocity and scalar fluctuations) for the four scalars and for horizontal velocity grouped into three stability classes

\begin{tabular}{llll}
\hline Stability parameter & Number of runs & $\begin{array}{l}\text { Integral time scale } \\
\Lambda(\mathrm{m})\end{array}$ & $\begin{array}{l}\text { Transport efficiency } \\
R_{w s}\end{array}$ \\
\hline Stable & $u: 3,072$ & $\Lambda_{u}: 55 \div 183$ & $R_{w u}=0.30 \div 0.40$ \\
$z / L>0.05$ & $T: 3,246$ & $\Lambda_{t}: 50 \div 129$ & $R_{w t}=0.28 \div 0.39$ \\
& $q: 3,212$ & $\Lambda_{q}: 42 \div 174$ & $R_{w q}=0.05 \div 0.15$ \\
& $c: 2,061$ & $\Lambda_{c}: 80 \div 215$ & $R_{w c}=0.11 \div 0.25$ \\
Near neutral & $m: 1,532$ & $\Lambda_{m}: 68 \div 168$ & $R_{w m}=0.01 \div 0.06$ \\
$|z / L| \leq 0.05$ & $u: 10,610$ & $\Lambda_{u}: 30 \div 83$ & $R_{w u}=0.37 \div 0.44$ \\
& $T: 10,533$ & $\Lambda_{t}: 37 \div 127$ & $R_{w t}=0.21 \div 0.37$ \\
& $q: 10,593$ & $\Lambda_{q}: 50 \div 151$ & $R_{w q}=0.09 \div 0.30$ \\
Unstable & $c: 8,951$ & $\Lambda_{c}: 98 \div 192$ & $R_{w c}=0.11 \div 0.27$ \\
$z / L<-0.05$ & $m: 1,522$ & $\Lambda_{m}: 60 \div 215$ & $R_{w m}=0.02 \div 0.06$ \\
& $u: 2,714$ & $\Lambda_{u}: 32 \div 86$ & $R_{w u}=0.35 \div 0.46$ \\
& $T: 2,811$ & $\Lambda_{t}: 24 \div 142$ & $R_{w t}=0.37 \div 0.45$ \\
& $q: 2,770$ & $\Lambda_{q}: 34 \div 120$ & $R_{w q}=0.34 \div 0.41$ \\
& $c: 1,862$ & $\Lambda_{c}: 35 \div 205$ & $R_{w c}=0.25 \div 0.35$ \\
& $m: 644$ & $\Lambda_{m}: 24 \div 176$ & $R_{w m}=0.02 \div 0.07$ \\
\hline
\end{tabular}

\subsection{The Inference of $\ell_{s}$ and $\tau_{s}$}

The two basic length and time scales, $\ell_{s}$ and $\tau_{s}$, used in the closure assumptions need to be estimated, at least for order of magnitude calculations. If the same eddies are producing scalar fluctuations and dissipating them at the same relaxation time scale, $\tau_{s}$ can be considered as a unique time scale for all the scalar concentration moments. Here, $\tau_{s}=\frac{\overline{s^{\prime 2}}}{\varepsilon_{s}}$ can be determined using the scalar variance dissipation rate derived from Kolmogorov's inertial subrange theory. For the experimental set-up here, this direct approach may prove difficult. One primary reason is that the sensors were positioned relatively close to the soil surface ( $3 \mathrm{~m}$ height), which resulted in less than one decade of inertial subrange scales for estimating dissipation rates before instrument path length averaging $(\sim 0.1 \mathrm{~m})$ and frequency cut-off $(10 \mathrm{~Hz})$ prohibited further analysis at finer scales (Katul et al. 1997). Other limitations include internal intermittency, known to increase the magnitude of the spectral slope (Warhaft 2000), the effects of large-scale contamination of inertial scales (Katul et al. 2003), and instrument-induced blue noise. An attempt to follow this approach produced results that contained low signal-to-noise ratio allowing unambiguous determination.

An alternative to the relaxation time scale is the integral time scale (Lenschow and Stankov 1986). While the former indicates how quickly an eddy is dissipated, the latter indicates how long an eddy remains coherent. So, it is likely that these two time scales are interdependent in the ASL, which is advantageous because the integral time scale can be computed readily from the area beneath the autocorrelation function. Table 1 presents the statistics of these time scales (transformed in length scale with the use of Taylor's hypothesis) for three broad stability classes (for all scalars and longitudinal velocity). When compared with longitudinal velocity, the length scales for scalars are generally larger and exhibit more variability.

The length scale $\ell_{s}$, related to the mean vertical gradients in scalar concentrations, is replaced here by the length scale associated with the peak of the cospectra. To perform this analysis, we use an ensemble of cospectra, first normalized to unit energy, and grouped into 

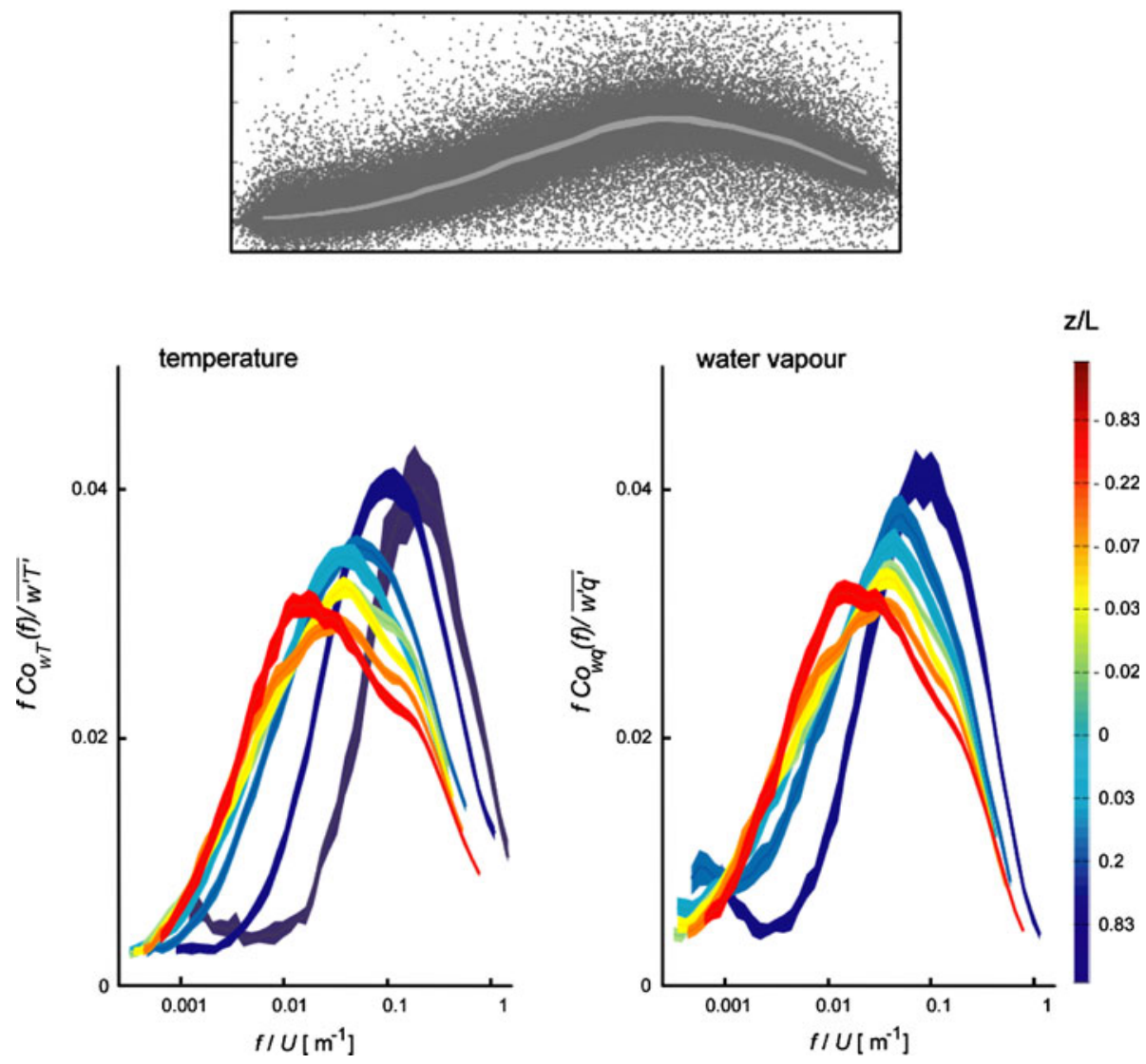

Fig. 6 Ensemble wavelet cospectra for temperature (left) and water vapour ( right). The cospectra are grouped based on stability classes ranging from slightly stable to slightly unstable (see colour bar). Coloured areas include 10\% (arbitrary chosen for graphical purposes) of the distribution of the observations for each length scale, and solid lines represent the median. The top panel shows one example of how the ensemble cospectrum is derived (grey dots represent the cospectrum for individual runs at a specific wavenumber)

different diabatic classes, as shown in Fig. 6. For each wavenumber and for each diabatic class, the median of several hundred individual cospectral densities was then computed. The positions of the peak shift one order of magnitude and the cospectra become narrower when passing from convective to stable conditions.

Figure 7 summarizes how the relative length scale corresponding to the peaks of the cospectra varies for a limited range of atmospheric stability conditions. As commonly assumed, approaching unstable conditions, eddies responsible for vertical transport become larger. Moreover, we find that the canonical shapes of the temperature and water vapour cospectra and their shifts with variations in atmospheric stability are in good agreement.

The basic features of the spectra are captured using a simple parametric model in the form: $S(\kappa)=S_{0}\left(1+\kappa / \kappa_{\mathrm{m}}\right)^{-\gamma}$, where $S_{0}, k_{\mathrm{m}}$ and $\gamma$ are fitting parameters. The results of the fitting are reported in Table 2 for all scalars and longitudinal velocity. The asymptotic slope $\gamma$ is greater than $5 / 3$, which relates to the Kolmogorov slope, even for the longitudinal velocity spectra. 
Fig. 7 Variations of the characteristic length scales corresponding to the peak of the cospectra (obtained from Fig. 6) as a function of the atmospheric stability parameter $(z / L)$ for temperature (dotted line) and water vapour (solid line)

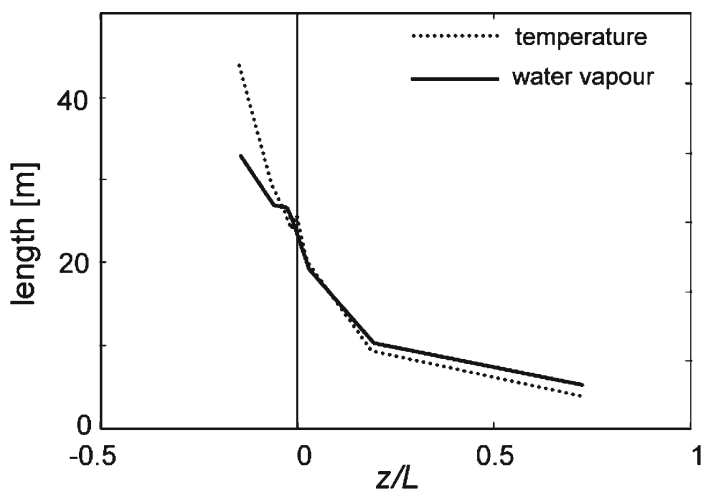

\section{Discussion}

It was demonstrated that, in the absence of exogenous effects, $v_{s}^{-1} u_{*}$ represents the endogenous (or equilibrium) scaling behaviour of scalar moments of any order. To explore how the exogenous factors disturb scalar concentration fluctuations, Fig. 8 presents $\sqrt{v_{s}^{-1} u_{*}}(=$ $\sigma_{s} / s_{*}$ ) for the entire dataset computed from normalized standard deviations (often used in flux-variance similarity studies).

The expression derived in Fig. 4, presumably representing the endogenous scaling, is also shown in Fig. 8 and evidently represents a lower envelope for the entire data. The proposed simplified scaling is clearly not applicable to all conditions and fails even for temperature when approaching adiabatic conditions. For such conditions, the $\sigma_{T} / T_{*}$ values are known to diverge from MOST. These runs are associated with near dawn/dusk periods, when surface heating/cooling is weak and conditions are non-stationary (e.g., Smedman et al. 2007). Methane does not exhibit any preferential scaling, neither during unstable nor during stable conditions. This is not surprising because, as earlier stated, the soil was a strong source of methane (i.e. there is no clear 'scalar boundary layer' in which $m_{*}$ is a scaling variable) and much of the variance is likely to be attributed to advective effects from cattle emissions. These types of sources are heterogeneous because cattle are grouped in clusters, and non-stationary because herbivorous species are itinerant.

We cannot exclude the fact that methane fluctuations observed at night may have originated from surrounding areas that are potentially active in methanogenesis (flooded fields, drainage ditches, marshlands, etc.) or by other non-local effects (e.g., collapsing ABL). As already stated, during stable stratification, the ABL is shallow, mixing is weak, concentrations can increase rapidly (Salmond and McKendry 2005) and tracers can be advected without much 'smearing' by turbulence. In this case, the use of $m_{*}$ as a representative concentration scaling parameter, is not appropriate. However, greater variances are still related to higher fluxes and lower friction velocities, as stated by MOST, but without any significant correlation because they are influenced by a random event (such as the passage of a cow) that is independent of microclimatic conditions.

For the remaining scalars, better similarity was noted during unstable conditions than during stable conditions. This again agrees with the idea that the unstable ASL appears relatively insensitive to ground source inhomogeneities, due to larger eddy sizes and more intense turbulence enhanced by positive buoyancy flux (Figs. 6, 7). The quantity $\sigma_{c} / c_{*}$ shows large scatter when compared to $\sigma_{q} / q_{*}$, but unlike methane it remains bounded at the lower end by 


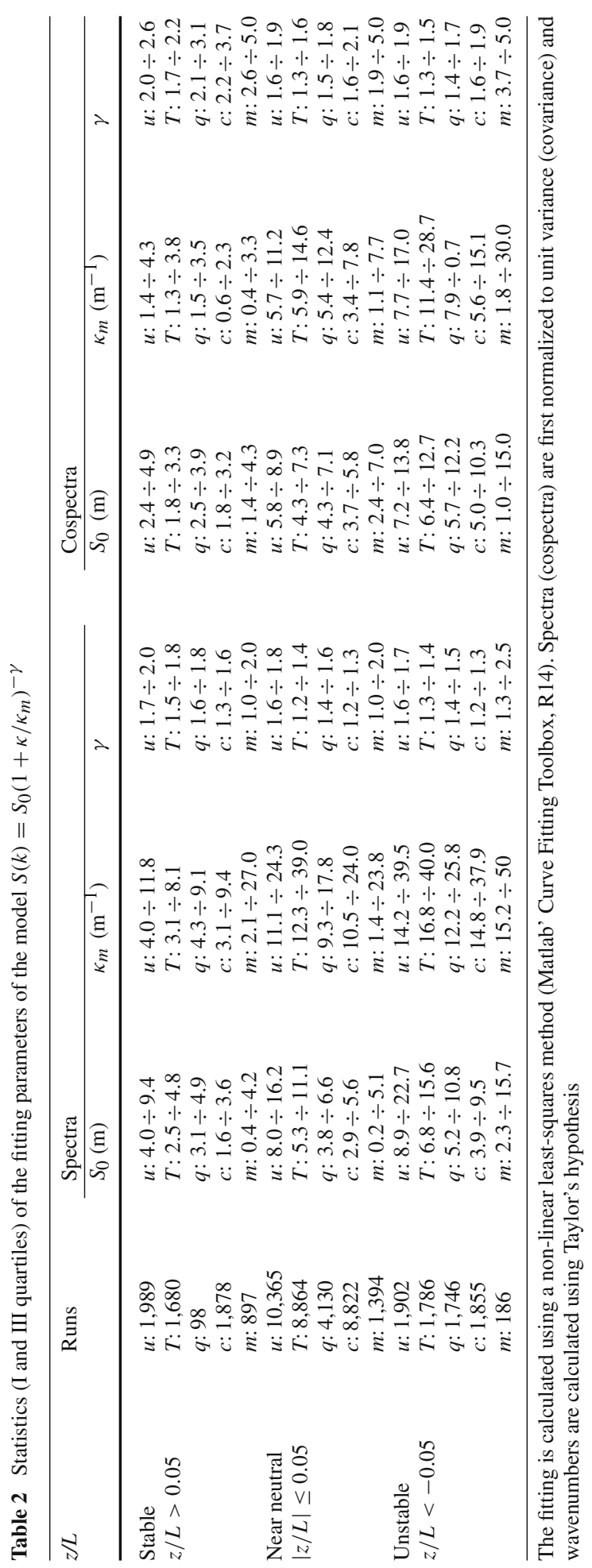



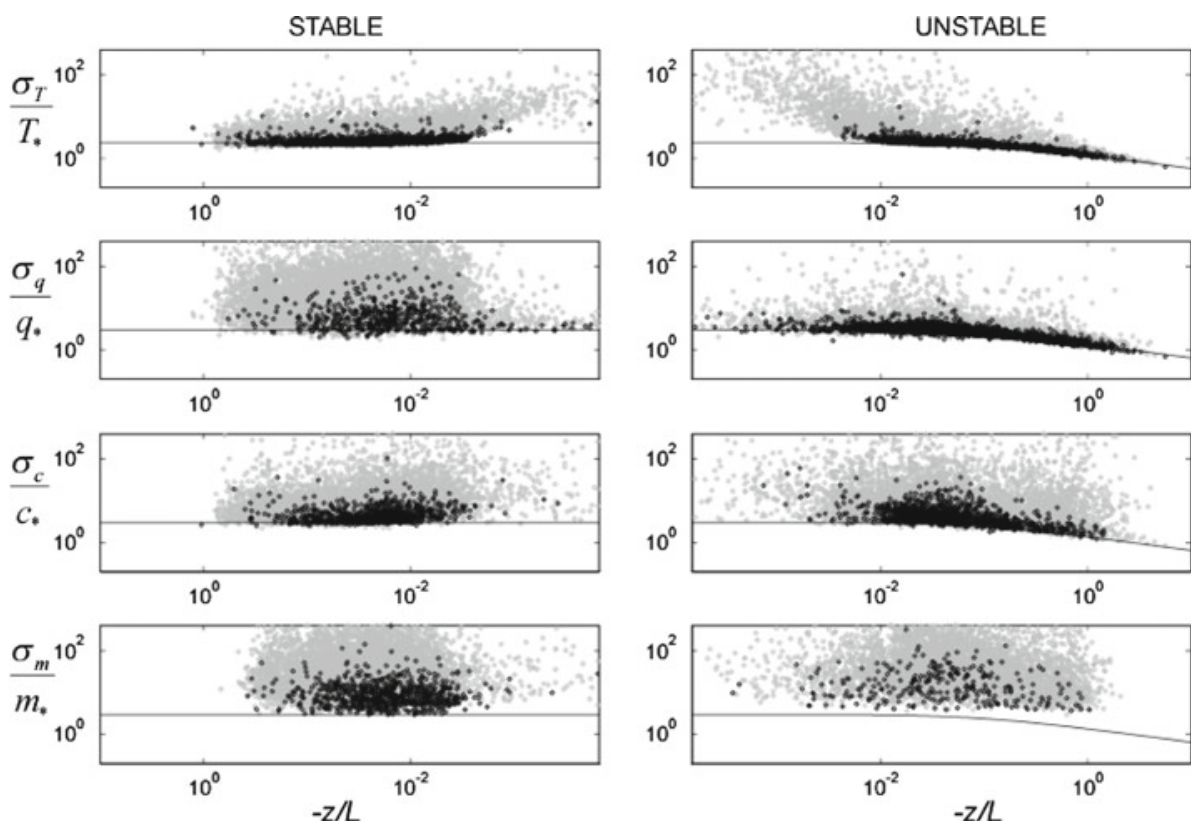

Fig. 8 Normalized standard deviations for the four scalar fluctuations for stable (left) and unstable (right) atmospheric stability conditions. Runs with storage term $z \frac{\partial \bar{s}}{\partial t}<0.005 \overline{w^{\prime} s^{\prime}}$ are shown as black dots. These runs follow more closely MOST scaling

MOST scaling (Fig. 8). In general, for any scalar, we can conclude that exogenous effects cannot be excluded.

\subsection{Can the Global Concentration Statistics be Decomposed into Exogenous and Endogenous Effects?}

A logical starting point for exploring the statistics of these exogenous effects is to assume that MOST scaling represents the 'endogenous' or 'equilibrium' state as earlier noted. When mass is injected from the ground into the ASL, its global scaling properties are strictly described by $v_{s}$. Exogenous effects may be viewed as additional disturbances that lead to variability over and above MOST scaling (as evidenced by the budget equations $3 \mathrm{a}$ and $3 \mathrm{~b}$ ), even under otherwise ideal micrometeorological conditions, e.g., an extensive fetch, a flat site and high winds. This 'extra' variance attributed to exogenous factors has already emerged in several previous studies (Katul et al. 1995; Pahlow et al. 2001; Lamaud and Irvine 2006; Cava et al. 2008; Detto et al. 2008). Motivated by these results, Eq. 4 may be (heuristically) written as

$$
\overline{\overline{s^{\prime n}}}=\frac{s_{*}}{v_{s}}+\frac{\overline{s_{N-L}^{\prime n}}}{\overline{w s^{\prime n-1}}},
$$

where the subscript $N-L$ indicates fluctuations originating from non-local, exogenous factors (to include all terms neglected in Eq. 3b). A crucial assumption in Eq. 5 is that scalar disturbances do not affect vertical fluxes, only scalar variances. This assumption is not entirely heuristic. 

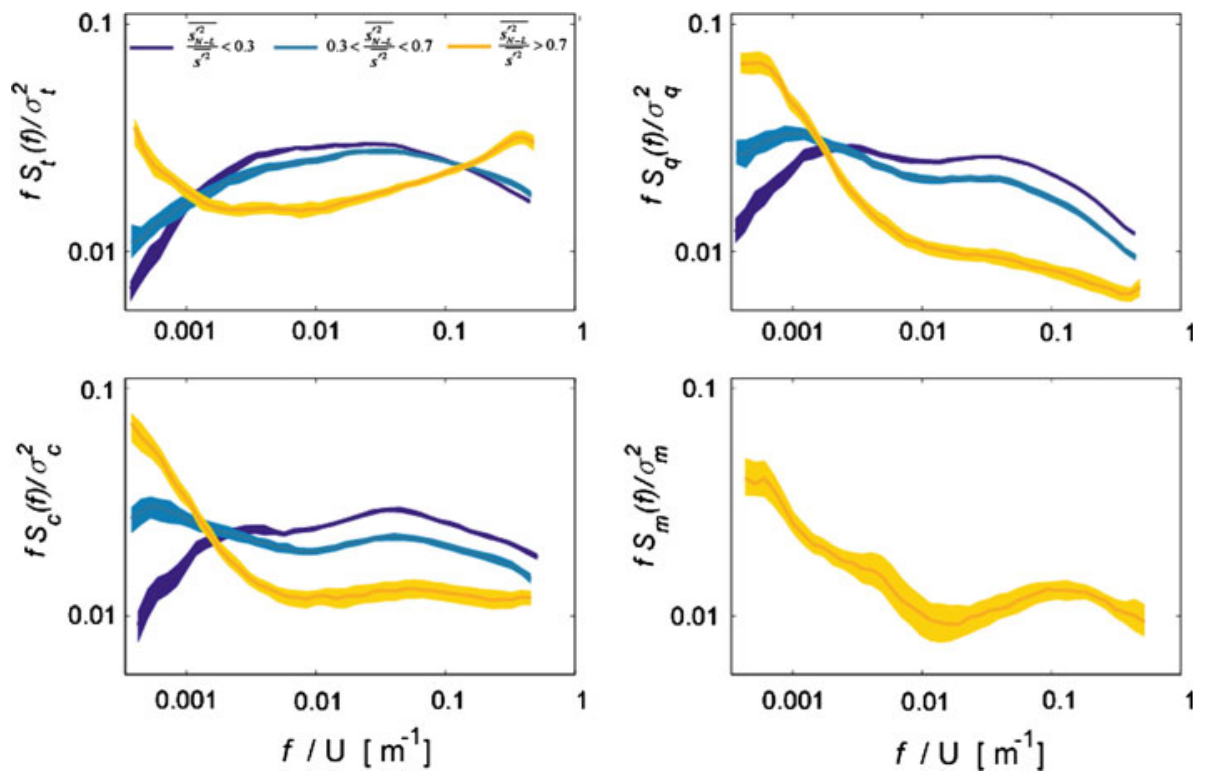

Fig. 9 Ensemble-averaged normalized wavelet spectra as a function of wavenumber inferred from Taylor's frozen turbulence hypothesis. The spectra are grouped for different value of $\overline{s_{N-L}^{\prime 2}} / \overline{s^{\prime 2}}$. Coloured areas include the middle $10 \%$ of the probability distribution function of the spectra for each group

The passive characteristic of these scalars ensures that, once a tracer from an exogenous source is emitted into the atmosphere, its trajectories follow the same statistics as all other tracers emitted from ground sources that are homogeneous and stationary. This implies that the fluctuations imposed by the flow, and in particular along the vertical axis, are the same for all these scalars originating from planar homogeneous and stationary emissions. These exogenous effects can also be characterized by frequencies lower than those responsible for turbulent vertical transport (e.g., unsteadiness). However, the dynamics of the ASL preclude the generation of large vertical velocity scales so that, if exogenous effects do produce fluxes, it would be at smaller scales. When compared to their scalar variance counterpart, the low frequency contribution of the vertical transport should be less appreciable.

We quantitatively test this assumption searching for the signature of the exogenous factors in the ensemble spectra and cospectra, with the same method previously adopted to generate the ensemble statistic. For illustration, this analysis is limited to near-neutral cases, where $|z / L|<0.05$. The ensemble spectra (computed and shown in Fig. 9) are conditionally grouped for each scalar, according to the relative importance of the non-local variance, $\overline{s_{N-L}^{\prime 2}} / \overline{s^{\prime 2}}$. The low frequency regions of the scalar power spectra show that individual contributions at these large scales can be significant, as large as or even larger than contributions from turbulent scales. Interestingly, the temperature spectrum for high values of the non-local variance suggests that the long wavelength contribution is not necessarily connected to the turbulent scales (which seem to be inactive or covered by instrumental noise). As we see later, even though these runs may be associated with small sensible heat fluxes, given that this analysis is restricted to $|z / L|<0.05$, the correlations with vertical velocity are still significant.

For other classes, low frequency components of temperature spectra decay rapidly. $\mathrm{CO}_{2}$ and $\mathrm{H}_{2} \mathrm{O}$ spectra, on the other hand, show two characteristic 'humps': the first peak is 

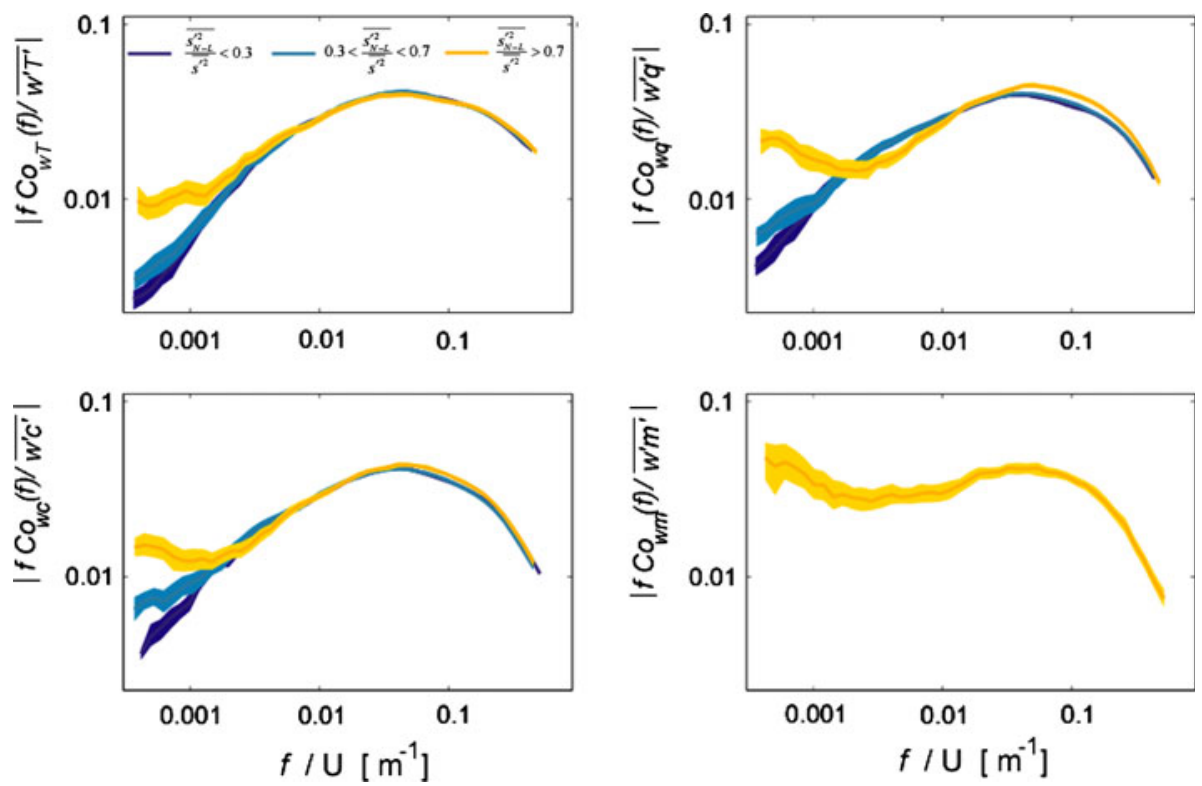

Fig. 10 Same as Fig. 9 but for the scalar cospectral magnitude. Note the insensitivity of the turbulent scales of the scalar cospectra to $\overline{s_{N-L}^{\prime 2}} / \overline{s^{\prime 2}}$

localized at turbulent scales (around $30 \mathrm{~m}$ ) and the second around $500 \mathrm{~m}$, a length comparable to the extension of the paddock (see Fig. 1). For methane concentration, a reliable number of spectra for the first two classes were not available (as expected from Fig. 8) given also the large noise contribution on small fluxes when the cattle were not present within the footprint. However, the shape of the spectra for the last class, when non-local effects dominate variance production, appears consistent with the other two scalars and with a clear peak at turbulent scales.

The cospectra also reveal minor activity at low frequencies, but their contribution is generally small when compared to the pronounced peaks at the turbulent scales (Fig. 10). When combined together, the results in Figs. 8 and 9 support the argument that exogenous effects tend to weaken the vertical transport efficiency, defined as the correlation coefficient $R_{w s}=\overline{w^{\prime} s^{\prime}} /\left(\sigma_{w} \sigma_{s}\right)$, primarily by increasing the scalar variance, $\sigma_{s}$, rather than reducing $\overline{w^{\prime} s^{\prime}}$. Depending on the atmospheric stability, typical values of $R_{w s}$ in the ASL range from 0.35 to 0.45 (Kaimal and Finningan 1994). Measured values of $R_{w s}$, reported in Table 1, are clearly underestimated, and not because of the vertical velocity statistics, which follow $\mathrm{M}-\mathrm{O}$ scaling (not shown).

Note that, in Fig. 10, the energy at lower frequencies appears positive because the magnitude of the cospectra is displayed here. The ensemble scalar flux cospectra assume a characteristic 'sickle shape' with a detectable gap. Vickers and Mahrt (2006) and Voronovich and Kiely (2007) exploited this scale gap to filter cospectra, taking advantage of the linearity in the Reynolds averaging operator. However, this scale separation (in the ensemble) is more difficult to detect for each individual cospectral run.

The size of the averaging window required to 'cut-off' low frequency motion is on the order of 2-4 $\min (500-1,000 \mathrm{~m}$, from Fig. 10), which is comparable to the integral time scales (Table 1). For the particular case of methane, where the exogenous factors are represented 
by cattle, the power of the cospectra at lower frequencies is comparable to the turbulent power. Surprisingly, the turbulent peak is at the same scale as all other scalar spectra (around 15-25 m). At higher frequencies, all other cospectra, especially methane, decay more rapidly than temperature, probably because attenuation effects related to the use of open- or closed-path gas analyzers (Massman 2000).

The picture that emerges from this comparative spectral and cospectral analysis shows that, in the ASL, exogenous effects on concentration statistics are connected to low frequency regions and are more persistent in scalar amplitude fluctuations when compared to the scalar fluxes (consistent with the assumption in Eq. 5). The spectral analysis of higher-order fluctuations shows similar patterns. The spectral representations for the third and fourth moments (which can be computed from the wavelet coefficients $W T(f)$ as $\frac{|W T(f)|^{3}}{\overline{s^{\prime 3}}}$ and $\frac{|W T(f)|^{4}}{\overline{s^{\prime 4}}}$ ) show how the relative power in the lower frequencies increases as exogenous effects become more significant (not shown).

\subsection{What is the Ensemble Signature of the Primary Exogenous Effect?}

Guided by the analysis in Fig. 5 that $v_{s}^{-1} u_{*}$ describes well the scaling properties of the higher-order moments when storage fluxes are conditionally removed, we explore here the role of the storage and advection terms (for the 2 months with measurements at two levels) in explaining the ensemble effects of the exogenous factors. When time series are only available at a single point, a correct evaluation of the storage term is not possible. The storage term is the difference between the instantaneous spatially-averaged profiles at the beginning and at the end of a time-averaging period. Commuting space with time usually invokes ergodicity, which requires stationarity. Hence, two simplifications are used to evaluate storage fluxes. First, it is assumed that the term $\partial \bar{s} / \partial t$ does not vary appreciably with height (which was shown to be reasonable for the 2 months of available data at two levels). Second, the instantaneous spatially-averaged mean concentration is replaced with its temporally-averaged counterpart over a sub-interval centred at the beginning and at the end of the averaging period (Finnigan 2006). This sub-interval needs to be long enough to capture a representative number of eddies. We use a 4-min averaging window because it is small enough to define multiple sub-intervals but larger than the scalar integral time scales (Table 1). In Fig. 11, the ensemble-averaged storage term (assumed to converge to the spatial average) is presented as a function of time of day. It is evident that the magnitude of the storage terms is related to the temporal dynamics of the sources. For $T$ and $c$, the storage terms became important only at dawn and dusk, when the sources switch sign and mean concentrations change rapidly (as expected for such short vegetation). For both scalars, this switching is more important at dusk (diurnal asymmetry). However, for long-term averaging, this term probably cancels out. During daytime, the scalar storage flux is overall negligible except for $m$, probably because even slow changes in concentrations are associated with small fluxes.

In this particular ecosystem, all scalars appear to respond to a degree of non-locality, which becomes more significant when surface fluxes are weak compared to their disturbances.

Quantification of these effects with single tower measurements, in relation to eddy-covariance applications for example, becomes problematic. Using two months of data available with a dual system, the vertical flux divergence can be computed. With two identical systems, and data processed in identical manner, differences in the fluxes between the two levels can be attributed to random measuring noise, systematic errors that depend on the height of the measurements, and finally flux divergence. Despite the relatively short vertical separation distance, about $1.5 \mathrm{~m}, \partial \overline{w^{\prime} s^{\prime}} / \partial z$ was not negligible for all scalars (methane, which 

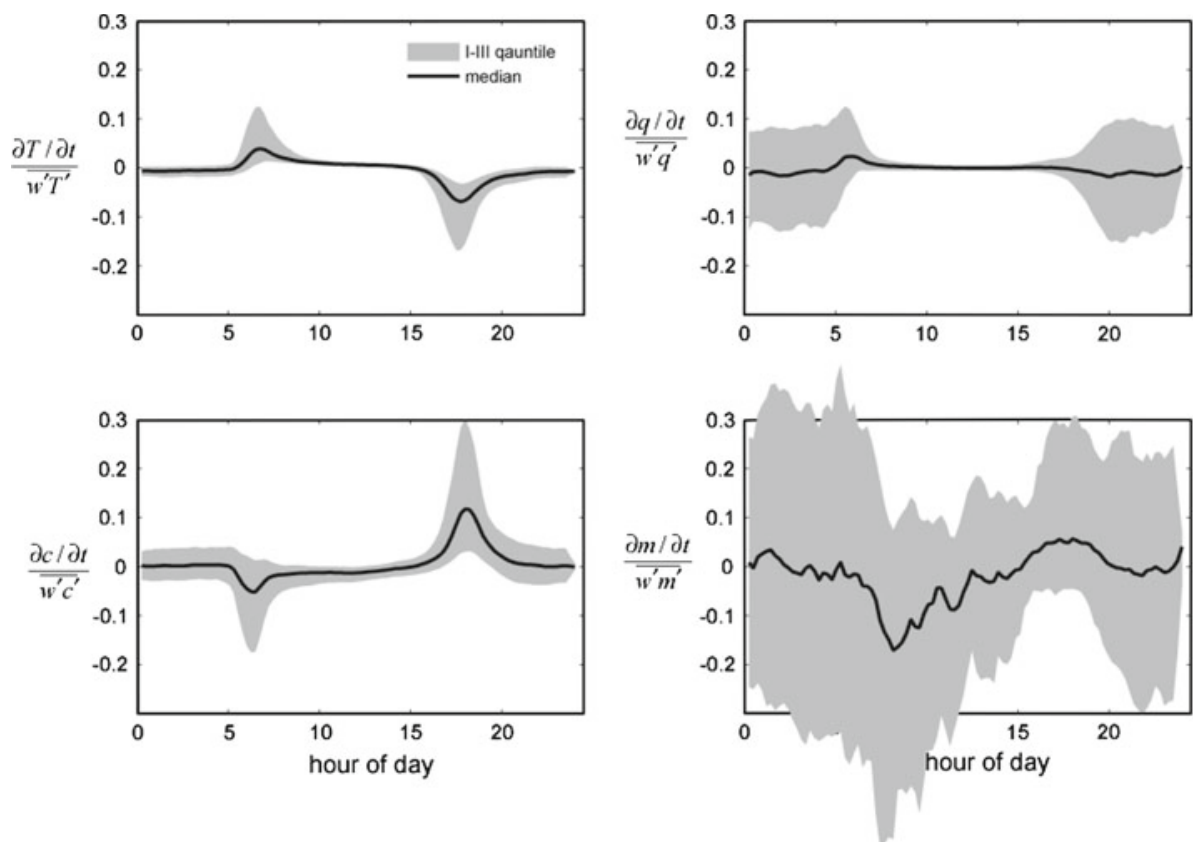

Fig. 11 Ensemble-averaged normalized storage flux as a function of time of day. Black lines are the median and the grey area bounds I and III quartiles of the statistics for each time of day

was not measured) as shown in Fig. 12. Ensemble averaging over the time of day to reduce the random errors, flux divergences may account for up to $10 \%$ of the total flux for $\mathrm{H}_{2} \mathrm{O}$ and $\mathrm{CO}_{2}$, and less so for temperature. More importantly, $\partial \overline{w^{\prime} s^{\prime}} / \partial z$ was poorly correlated with scalars $\left(R^{2}=0.23, p\right.$-value $<10^{-3}$ for temperature and not significantly correlated for $\mathrm{H}_{2} \mathrm{O}$ and $\mathrm{CO}_{2}$ ) and much smaller in magnitude than $\partial \bar{s} / \partial t$. From Eq. 1, this implies a significant contribution from the advection term $\bar{u}(\partial \bar{s}) /(\partial z)$ (given the coordinate transformation, $\bar{w}=0$ at both measuring heights). The lack of correlation between $\partial \overline{w^{\prime} s^{\prime}} / \partial z$ and $\partial \bar{s} / \partial t$ emphasises that advection is present also in steady-state conditions and is difficult to capture from single-point time series. Actually, in a heterogeneous landscape, advection may increase when the contrast in surface fluxes between the heterogeneous patches is large. This is evident in the water vapour flux, with the largest flux divergence occurring in the central hours of the day, when available energy is maximum but water vapour concentration is relatively steady in time (compare Figs. 11 and 12).

High frequency spectral flux attenuation decreases with height in neutral or unstable conditions (Horst 1997; Massman 2000). The fluxes of $\mathrm{H}_{2} \mathrm{O}$ and $\mathrm{CO}_{2}$, instead, reveal during the daytime exactly the opposite, so we can exclude attributing the scalar flux differences to systematic errors depending on height (such differences relate to high frequency cospectral losses).

During the day, the behaviour of $c$ is expected to be similar to $q$, but at night $\mathrm{CO}_{2}$ emissions are important and turbulent mixing acts differently on scalar dispersion. Carbon fluxes are complicated further by the fact that sinks during the day may not correspond to sources at night.

The storage term in the ASL appears to be more connected to the imbalance between production and dissipation in Eq. 2, rather than to flux divergence in the mean 

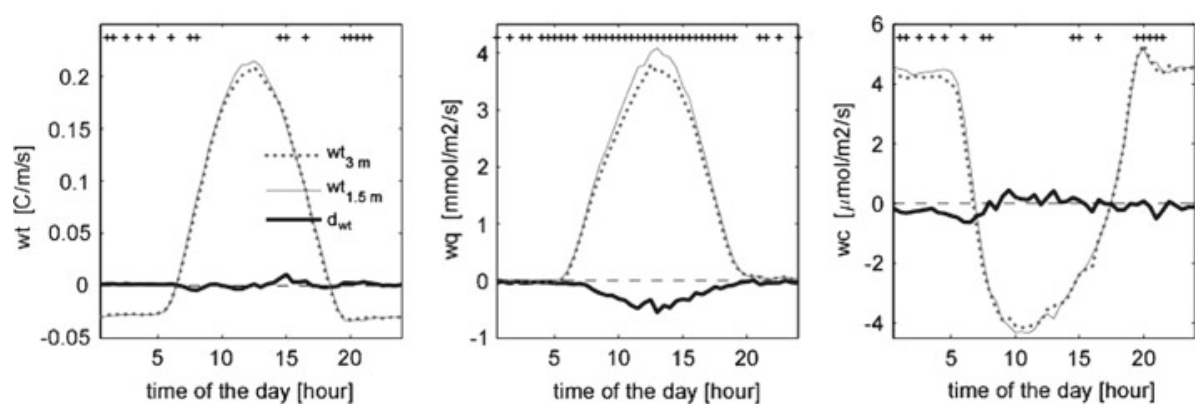

Fig. 12 Ensemble-averaged fluxes and flux divergence as a function of time of day for temperature, water vapour and carbon dioxide recorded during July-August 2009 (2600 runs). Plus signs on the top of each plot indicate hours when the null hypothesis that two samples derive from distributions with equal means $(t$-test with significance level $=0.05$ ) can be rejected

continuity equation (1). This is why it acts as a reasonable discriminator for the $n$-order MOST scaling (Figs. 3, 8), which implies that changes in mean concentration fields (first-order non-stationarity) are probably associated with large variations in the fluctuation field (second-order non-stationarity). However this condition is not sufficient to exclude exogenous effects, as shown by the large scatter still present in the MOST relations in Fig. 8.

\section{Conclusions}

The drained peatland observational area consisted of flooded and non-flooded fields that created a complex patchwork of mosaics with differences in source/sink spatial distributions of heat, water vapour, carbon dioxide and methane. In addition, the presence of cattle introduced an element of disturbance for some scalars $\left(\mathrm{CO}_{2}\right.$ and especially $\left.\mathrm{CH}_{4}\right)$ while absent for others. We examined how this patchwork, for an otherwise ideal micrometeorological site, affects scalar fluctuation statistics and turbulent fluxes a few metres above the soil. The scalars were shown to be incompletely blended by turbulence and to carry the signature of the source heterogeneity. Less turbulence (nocturnal boundary layer) and sharper discontinuities (irrigation ditches) lead to increased variability in scalar concentration fluctuations over and above that expected from a stationary and planar homogeneous state (referred to as the equilibrium state). These 'exogenous' effects act on length scales larger than those responsible for vertical scalar mass transport, and affect scalar concentration statistics when averaged over 20-60 min. Fortunately, for the eddy-covariance approach, vertical fluxes appear less affected by these factors because the dynamics of the boundary layer suppress long wavelength contributions in the vertical velocity fluctuations. However, other micrometeorological methods, which are not based on direct measurements of the fluxes, such as the flux-variance (FV) and the flux-gradient (FG) methods (or other methods that implicitly or explicitly make use of MOST as is the case in scintillometer or dissipation methods), need to be carefully interpreted. For example, for the FV method, which has also been suggested recently as the basis for partitioning flux components (Scanlon and Kustas 2010), we have shown that the scalar variances are much larger than those predicted from the equilibrium state thereby resulting in unrealistically high surface fluxes (the methane case here is an extreme example). For the FG method, we have shown that the scalar stability correction functions (inferred from correlation coefficients) may differ across scalars. 
Hence, inferring methane fluxes using an eddy-diffusivity similarity between methane and other scalars (e.g. heat or water vapour as is commonly done) becomes problematic for such a site. Moreover, while the FV method is more sensitive to non-stationarity, the FG method is more affected by heterogeneity effects. For the latter, if the vertical separation of the two levels is kept relatively large because expected vertical gradients are small, the footprint of the higher level is greater than the lower level, thereby exasperating the problem at this site.

These findings may have other implications when modelling scalar dispersion within the ASL, where higher-order statistics become important. One example is odour pollution and odour remediation methodologies, which necessitates understanding higher-order concentration statistics (Schiffman et al. 2005). Another one would be the intensity of segregation in multi-species chemical transport problems - in which the variances and the mean quantities determine whether a chemical scalar can be treated as passively transported by turbulence or not.

Acknowledgements We wish to thank Ted Hehn and Joe Verfaillie for their the support in the experimental set-up and maintenance, Yit Arn Teh for chamber measurements, Whendee Silver for advices on methane and carbon dioxide emissions, Dimmie Hendriks for useful discussions on managed peatland and Douglas Bear from Los Gatos Research for all the technical supports on the tunable laser. This research was supported by the National Science Foundation (award number NSF-ATM 06-28720). G. Katul acknowledges support from the National Science Foundation (NSF-EAR 06-35787, NSF-EAR-06-28432, and NSF-ATM-0724088), and the Binational Agricultural Research and Development (BARD, Research Grant No. IS3861-06).

Open Access This article is distributed under the terms of the Creative Commons Attribution Noncommercial License which permits any noncommercial use, distribution, and reproduction in any medium, provided the original author(s) and source are credited.

\section{References}

Acevedo OC, Moraes OLL, Fitzjarrald DR, Sakai RK, Mahrt L (2007) Turbulent carbon exchange in very stable conditions. Boundary-Layer Meteorol 125(1):49-61

Antonia RA, Smalley RJ (2001) Scaling range exponents from X-wire measurements in the atmospheric surface layer. Boundary-Layer Meteorol 100(3):439-457

Antonia RA, Chambers AJ, Bradley EF (1982) 3rd-order and 4th-order mixed moments of turbulent velocity and temperature-fluctuations in the atmospheric surface-layer. Boundary-Layer Meteorol 22(4):421-430

Argoul F, Arneodo A, Grasseau G, Gagne Y, Hopfinger EJ, Frisch U (1989) Wavelet analysis of turbulence reveals the multifractal nature of the Richardson cascade. Nature 338(6210):51-53

Asanuma J, Brutsaert W (1999) The effect of chessboard variability of the surface fluxes on the aggregated turbulence fields in a convective atmospheric surface layer. Boundary-Layer Meteorol 91(1):37-50

Baer DS, Paul JB, Gupta JB, O'Keefe A (2002) Sensitive absorption measurements in the near-infrared region using off-axis integrated-cavity-output spectroscopy. Appl Phys B 75(2-3):261-265

Baldocchi DD (2003) Assessing the eddy covariance technique for evaluating carbon dioxide exchange rates of ecosystems: past, present and future. Glob Chang Biol 9(4):479-492

Baldocchi DD, Meyers TP (1988) A spectral and lag-correlation analysis of turbulence in a deciduous forest canopy. Boundary-Layer Meteorol 45(1-2):31-58

Bradley EF, Antonia RA, Chambers AJ (1981) Temperature structure in the atmospheric surface-layer. 1. The budget of temperature variance. Boundary-Layer Meteorol 20(3):275-292

Cava D, Giostra U, Siqueira M, Katul GG (2004) Organised motion and radiative perturbations in the nocturnal canopy sublayer above an even-aged pine forest. Boundary-Layer Meteorol 112(1):129-157

Cava D, Katul GG, Sempreviva AM, Giostra U, Scrimieri A (2008) On the anomalous behaviour of scalar flux-variance similarity functions within the canopy sub-layer of a dense alpine forest. Boundary-Layer Meteorol 128(1):33-57

Chu CR, Parlange MB, Katul GG, Albertson JD (1996) Probability density functions of turbulent velocity and temperature in the atmospheric surface layer. Water Resour Res 32(6):1681-1688

Cicerone RJ, Oremland RS (1988) Biogeochemical aspects of atmospheric methane. Glob Biogeochem Cycles 2:299-327 
De Bruin HAR, Van Den Hurk BJJM, Kroon LJM (1999) On the temperature-humidity correlation and similarity. Boundary-Layer Meteorol 93(3):453-468

Detto M, Katul GG (2007) Simplified expressions for adjusting higher-order turbulent statistics obtained from open path gas analyzers. Boundary-Layer Meteorol 122(1):205-216

Detto M, Montaldo N, Albertson JD, Mancini M, Katul GG (2006) Soil moisture and vegetation controls on evapotranspiration in a heterogeneous Mediterranean ecosystem on Sardinia, Italy. Water Resour Res 42(8):W08419

Detto M, Katul G, Mancini M, Montaldo N, Albertson JD (2008) Surface heterogeneity and its signature in higher-order scalar similarity relationships. Agric For Meteorol 148(6-7):902-916

Farge M (1992) Wavelet transforms and their applications to turbulence. Annu Rev Fluid Mech 24:395-457

Finnigan JJ (2006) The storage term in eddy flux calculations. Agric For Meteorol 136(3-4):108-113

Finnigan JJ (2008) An introduction to flux measurements in difficult conditions. Ecol Appl 18(6):1340-1350

Finnigan JJ, Einaudi F, Fua D (1984) The interaction between an internal gravity-wave and turbulence in the stably-stratified nocturnal boundary-layer. J Atmos Sci 41(16):2409-2436

Garratt JR (1992) The atmospheric boundary layer. Cambridge University Press, U.K., 316 pp

Hendriks DMD, Dolman AJ, Van Der Molen MK, van Huissteden J (2008) A compact and stable eddy covariance set-up for methane measurements using off-axis integrated cavity output spectroscopy. Atmos Chem Phys 8(2):431-443

Horst TW (1997) A simple formula for attenuation of eddy fluxes measured with first-order-response scalar sensors. Boundary-Layer Meteorol 82(2):219-233

Hsieh CI, Katul GG (1997) Dissipation methods, Taylor's hypothesis, and stability correction functions in the atmospheric surface layer. J Geophys Res Atmos 102(D14):16391-16405

Hsieh CI, Katul GG, Chi T (2000) An approximate analytical model for footprint estimation of scaler fluxes in thermally stratified atmospheric flows. Adv Water Resour 23(7):765-772

Hudgins L, Friehe CA, Mayer ME (1993) Wavelet transforms and atmospheric-turbulence. Phys Rev Lett 71(20):3279-3282

Kaimal JC, Finningan JJ (1994) Atmospheric boundary layer flows. Oxford University Press, New York, $289 \mathrm{pp}$

Kaimal JC, Gaynor JE (1991) Another look at sonic thermometry. Boundary-Layer Meteorol 56(4):401-410

Kaimal JC, Wyngaard JC (1990) The Kansas and Minnesota experiments. Boundary-Layer Meteorol $50(1-4): 31-47$

Katul GG, Goltz SM, Hsieh CI, Cheng Y, Mowry F, Sigmon J (1995) Estimation of surface heat and momentum fluxes using the flux-variance method above uniform and non-uniform terrain. Boundary-Layer Meteorol 74(3):237-260

Katul GG, Hsieh CI, Sigmon J (1997) Energy-inertial scale interactions for velocity and temperature in the unstable atmospheric surface layer. Boundary-Layer Meteorol 82(1):49-80

Katul GG, Vidakovic B, Albertson J (2001) Estimating global and local scaling exponents in turbulent flows using discrete wavelet transformations. Phys Fluids 13(1):241-250

Katul GG, Angelini C, De Canditiis D, Amato U, Vidakovic B, Albertson JD (2003) Are the effects of large scale flow conditions really lost through the turbulent cascade? Geophys Res Lett 30(4):1164

Katul GG, Sempreviva AM, Cava D (2008) The temperature-humidity covariance in the marine surface layer: a one-dimensional analytical model. Boundary-Layer Meteorol 126(2):263-278

Kiely G, Albertson JD, Parlange MB, Eichinger WE (1996) Convective scaling of the average dissipation rate of temperature variance in the atmospheric surface layer. Boundary-Layer Meteorol 77(3-4):267-284

Lamaud E, Irvine M (2006) Temperature and humidity dissimilarity and heat-to-water-vapour transport efficiency above and within a pine forest canopy: the role of the Bowen ratio. Boundary-Layer Meteorol 120(1):87-109

Lenschow DH, Stankov BB (1986) Length scales in the convective boundary-layer. J Atmos Sci 43(12): 1198-1209

Mahrt L (1989) Intermittency of atmospheric-turbulence. J Atmos Sci 46(1):79-95

Mahrt L, Sun J, Blumen W, Delany T, Oncley S (1998) Nocturnal boundary-layer regimes. Boundary-Layer Meteorol 88(2):255-278

Massman WJ (2000) A simple method for estimating frequency response corrections for eddy covariance systems. Agric For Meteorol 104(3):185-198

McNaughton KC (2006) On the kinetic energy budget of the unstable atmospheric surface layer. BoundaryLayer Meteorol 118(1):83-107

McNaughton KG, Laubach J (1998) Unsteadiness as a cause of non-equality of eddy diffusivities for heat and vapour at the base of an advective inversion. Boundary-Layer Meteorol 88(3):479-504

Monin AS, Obukhov AM (1954) Basic laws of turbulent mixing in the ground layer of the atmosphere. Trans Geophys Inst Akad Nauk USSR 151:163-187 
Monji N (1973) Budgets of turbulent energy and temperature variance in the transition zone from forced to free convection. J Meteorol Soc Jpn 51:133-145

Nappo CJ (1991) Sporadic breakdowns of stability in the PBL over simple and complex terrain. BoundaryLayer Meteorol 54(1-2):69-87

Nichols-Pagel GA, Percival DB, Reinhall PG, Riley JJ (2008) Should structure functions be used to estimate power laws in turbulence? A comparative study. Phys D 237(5):665-677

Pahlow M, Parlange MB, Porte-Agel F (2001) On Monin-Obukhov similarity in the stable atmospheric boundary layer. Boundary-Layer Meteorol 99(2):225-248

Panofsky H, Dutton J (1984) Atmospheric turbulence, models and methods for engineering applications. Wiley International, New York, 389 pp

Percival DP (1995) On estimation of the wavelet variance. Biometrika 82(3):619-631

Prabha TV, Leclerc MY, Karipot A, Hollinger DY (2007) Low-frequency effects on eddy covariance fluxes under the influence of a low-level jet. J Appl Meteorol Climatol 46(3):338-352

Salmond JA, McKendry IG (2005) A review of turbulence in the very stable nocturnal boundary layer and its implications for air quality. Prog Phys Geogr 29(2):171-188

Scanlon TM, Kustas WP (2010) Partitioning carbon dioxide and water vapor fluxes using correlation analysis. Agric For Meteorol 150(1):89-99

Schiffman SS, McLaughlin B, Katul GG, Nagle HT (2005) Eulerian-Lagrangian model for predicting odor dispersion using instrumental and human measurements. Sens Actuators B 106(1):122-127

Schotanus P, Nieuwstadt FTM, Debruin HAR (1983) Temperature-measurement with a sonic anemometer and its application to heat and moisture fluxes. Boundary-Layer Meteorol 26(1):81-93

Shaw SL et al (2007) Volatile organic compound emissions from dairy cows and their waste as measured by proton-transfer-reaction mass spectrometry. Environ Sci Technol 41(4):1310-1316

Shraiman BI, Siggia ED (2000) Scalar turbulence. Nature 405(6787):639-646

Smedman AS, Hogstrom U, Hunt JCR, Sahlee E (2007) Heat/mass transfer in the slightly unstable atmospheric surface layer. Q J Roy Meteorol Soc 133(622):37-51

Sreenivasan KR, Chambers AJ, Antonia RA (1978) Accuracy of moments of velocity and scalar fluctuations in the atmospheric surface layer. Boundary-Layer Meteorol 14(3):341-359

Stull RB (1988) An introduction to boundary layer meteorology. Kluwer, Dordrecht, 666 pp

Sun JL et al (2004) Atmospheric disturbances that generate intermittent turbulence in nocturnal boundary layers. Boundary-Layer Meteorol 110(2):255-279

Terradellas E, Morales G, Cuxart J, Yague C (2001) Wavelet methods: application to the study of the stable atmospheric boundary layer under non-stationary conditions. Dyn Atmos Oceans 34(2-4):225-244

Torrence C, Compo GP (1998) A practical guide to wavelet analysis. Bull Am Meteorol Soc 79(1):61-78

Townsend AA (1961) Equilibrium layers and wall turbulence. J Fluid Mech 11(1):97-120

Verma SB, Anderson DE (1984) Kolmogorov constants for CO2, wind velocity, air-temperature, and humidity fluctuations over a crop surface. Boundary-Layer Meteorol 28(1-2):161-167

Vickers D, Mahrt L (2006) A solution for flux contamination by mesoscale motions with very weak turbulence. Boundary-Layer Meteorol 118(3):431-447

von Randow C, Kruijt B, Holtslag AAM (2006) Low-frequency modulation of the atmospheric surface layer over Amazonian rain forest and its implication for similarity relationships. Agric For Meteorol 141(2-4):192-207

Voronovich V, Kiely G (2007) On the gap in the spectra of surface-layer atmospheric turbulence. BoundaryLayer Meteorol 122(1):67-83

Warhaft Z (2000) Passive scalars in turbulent flows. Annu Rev Fluid Mech 32:203-240

Wyngaard JC, Cote OR (1971) Budgets of turbulent kinetic energy and temperature variance in the atmospheric surface layer. J Atmos Sci 28(2):190-201

Zhang HS, Park SU (1999) Dissipation rates of turbulent kinetic energy and temperature and humidity variances over different surfaces. Atmos Res 50(1):37-51 